九州大学学術情報リポジトリ

Kyushu University Institutional Repository

\title{
dNLS Flow on Discrete Space Curves
}

\section{Hirose, Sampe i}

Center for Promotion of Educational Innovation, Shibaura Institute of Technology : Research Lecturer

Inoguchi, Jun-ichi

Institute of Mathematics, University of Tsukuba : Professor

Kajiwara, Kenji

Institute of Mathematics for Industry, Kyushu University : Professor

Matsuura, Nozomu

Faculty of Science, Department of Applied Mathematics, Fukuoka University : Assistant Professor

他

http://hdl. handle. net/2324/1547100

出版情報: MI lecture note series. 64, pp.93-102, 2015-09-18. Institute of Mathematics for Industry, Kyushu University

バージョン :

権利関係 : 


\title{
dNLS Flow on Discrete Space Curves
}

\author{
Sampei Hirose* \\ Center for Promotion of Educational Innovation, Shibaura Institute of Technology, \\ 307 Fukasaku, Minuma-ku, Saitama 337-8570, Japan \\ Jun-ichi Inoguchi ${ }^{\dagger}$ \\ Institute of Mathematics, University of Tsukuba, \\ Tsukuba, Ibaraki 305-8571, Japan \\ Kenji Kajiwara $^{\ddagger}$ \\ Institute of Mathematics for Industry, Kyushu University, \\ 744 Motooka, Nishi-ku, Fukuoka 819-0395, Japan \\ Nozomu Matsuura ${ }^{\S}$ \\ Department of Applied Mathematics, Fukuoka University, \\ Nanakuma 8-19-1, Fukuoka 814-0180, Japan \\ Yasuhiro Ohta \\ Department of Mathematics, Kobe University, \\ Rokko, Kobe 657-8501, Japan
}

\begin{abstract}
The local induction equation, or the binormal flow on space curves is a well-known model of deformation of space curves as it describes the dynamics of vortex filaments, and the complex curvature is governed by the nonlinear Schrödinger equation (NLS). In this paper, we present its discrete analogue, namely, a model of deformation of discrete space curves by the discrete nonlinear Schrödinger equation (dNLS). We also present explicit formulas for both NLS and dNLS flows in terms of the $\tau$ function of the 2-component KP hierarchy.
\end{abstract}

Keywords: space curves, discrete space curves, nonlinear Schrödinger equation, discrete nonlinear Schrödinger equation, integrable systems, $\tau$ functions, solitons

\section{Introduction}

The local induction equation (LIE)

$$
\frac{\partial \gamma}{\partial t}=\frac{\partial \gamma}{\partial x} \times \frac{\partial^{2} \gamma}{\partial x^{2}}
$$

is one of the most important models of deformation of space curves, where $\gamma(x, t) \in \mathbb{R}^{3}$ is a smooth space curve parametrized by the arc-length $x$ and $t$ is a deformation parameter. In a physical setting,

\footnotetext{
*hirose3@ shibaura-it.ac.jp

†inoguchi@math.tsukuba.ac.jp

${ }_{\ddagger}$ kaji@imi.kyushu-u.ac.jp

§nozomu@fukuoka-u.ac.jp

ฯohta@math.kobe-u.ac.jp
} 
it describes the dynamics of vortex filaments driven by the self-induction in the inviscid fluid under the local induction approximation [6].

It is well-known that if $\gamma$ obeys LIE, then the curvature and the torsion, or equivalently, the complex curvature of $\gamma$ solves the nonlinear Schrödinger equation (NLS) which is one of the most typical equations in the integrable systems. To show this, we use the Frenet frame $\Phi=\Phi(x, t)=$ $[T(x, t), N(x, t), B(x, t)] \in \mathrm{SO}(3)$, where $T, N, B$ are the tangent, the normal, and the binormal vectors defined by

$$
T=\gamma^{\prime}, \quad N=\frac{\gamma^{\prime \prime}}{\left|\gamma^{\prime \prime}\right|}, \quad B=T \times N, \quad{ }^{\prime}=\frac{\partial}{\partial x},
$$

respectively. Note that it follows that $|T|=\left|\gamma^{\prime}\right|=1$ since $x$ is the arc-length. Then we have the Frenet-Serret formula

$$
\frac{\partial \Phi}{\partial x}=\Phi L, \quad L=\left[\begin{array}{ccc}
0 & -\kappa & 0 \\
\kappa & 0 & -\lambda \\
0 & \lambda & 0
\end{array}\right],
$$

where $\kappa=\left|\gamma^{\prime \prime}\right|$ and $\lambda=-\left\langle B^{\prime}, N\right\rangle$ are the curvature and the torsion, respectively. In this setting, LIE (1) is expressed as the deformation by the binormal flow

$$
\frac{\partial \gamma}{\partial t}=\kappa B
$$

and the corresponding deformation equation of the Frenet frame is given by

$$
\frac{\partial \Phi}{\partial t}=\Phi M, \quad M=\left[\begin{array}{ccc}
0 & \kappa \lambda & -\kappa^{\prime} \\
-\kappa \lambda & 0 & -\frac{\kappa^{\prime \prime}}{\kappa}+\lambda^{2} \\
\kappa^{\prime} & \frac{\kappa^{\prime \prime}}{\kappa}-\lambda^{2} & 0
\end{array}\right] .
$$

The compatibility condition of the system of linear partial differential equations (3) and (5)

$$
\frac{\partial L}{\partial t}-\frac{\partial M}{\partial x}=L M-M L
$$

yields

$$
\frac{\partial \kappa}{\partial t}=-2 \frac{\partial \kappa}{\partial x} \lambda-\kappa \frac{\partial \lambda}{\partial x}, \quad \frac{\partial \lambda}{\partial t}=\frac{\partial}{\partial x}\left(\frac{\kappa^{\prime \prime}}{\kappa}+\frac{\kappa^{2}}{2}-\lambda^{2}\right) .
$$

Introducing the complex curvature $u=u(x, t) \in \mathbb{C}$ by the Hasimoto transformation[6]

$$
u=\kappa e^{\sqrt{-1} \Lambda}, \quad \Lambda=\int^{x} \lambda d x
$$

we see that $u$ satisfies NLS

$$
\sqrt{-1} \frac{\partial u}{\partial t}+\frac{\partial^{2} u}{\partial x^{2}}+\frac{1}{2}|u|^{2} u=0 .
$$

Also, one can show that this deformation is isoperimetric, namely $\left|\gamma^{\prime}\right|=1$ for all $t$.

Discretization of curves and their deformations preserving underlying integrable structures is an important problem in the discrete differential geometry. For example, the isoperimetric deformation of plane discrete curves described by the discrete $\mathrm{mKdV}$ equation $(\mathrm{dmKdV})$ has been studied in $[12,14,15]$. For discrete space curves, the deformations by the discrete sine-Gordon equation (dsG) and dmKdV has been studied in $[4,13,14]$, and the deformation by dNLS is formulated in $[11,18]$.

The dsG and dmKdV describe torsion-preserving isoperimetric and equidistant deformation of the space discrete curves with constant torsion. However, formulation of discrete deformation of space discrete curves with varying torsion is a difficult problem. The only example so far is presented by Hoffmann $[10,11]$, where he has claimed that composition of certain two isoperimetric equidistant 
deformations can be regarded as a discrete analogue of LIE. Also, it was used for numerical simulation of fluid flow $[18,19]$. This formulation uses quarternions and its geometric meaning is clear, but description of the deformation parameters in terms of the complex curvature, thus the relation to dNLS are rather indirect.

In this paper, we present a formulation of the dNLS flow on space discrete curves from different approach; the deformation of curves is expressed in terms of the discrete Frenet frame with the coefficients given by the curvature and torsion of the curves explicitly. In this approach, dNLS arises as the equation governing the complex curvature of curves, which is the same as the case of smooth curves. Based on this formulation, we present explicit formulas for the NLS flow for smooth curves and the dNLS flow to discrete curves in terms of $\tau$ functions of the two-component KP hierarchy by applying the theory of integrable systems. We expect that our dNLS flow can be an alternative to Hoffmann's formulation when it is used to simulate the dynamics of fluids. Also, explicit expression of the scheme and exact solutions may promote further development of theoretical studies of discrete dynamics of discrete curves from both mathematical and physical point of view.

\section{Deformations of space curves}

Let $\gamma_{n} \in \mathbb{R}^{3}$ be a discrete space curve with

$$
\left|\gamma_{n+1}-\gamma_{n}\right|=\epsilon,
$$

where $\epsilon$ is a constant. We introduce the discrete Frenet frame $\Phi_{n}=\left[T_{n}, N_{n}, B_{n}\right] \in \mathrm{SO}(3)$ by

$$
T_{n}=\frac{\gamma_{n+1}-\gamma_{n}}{\epsilon}, \quad B_{n}=\frac{T_{n-1} \times T_{n}}{\left|T_{n-1} \times T_{n}\right|}, \quad N_{n}=B_{n} \times T_{n} .
$$

Then it follows that the discrete Frenet frame satisfies the discrete Frenet-Serret formula

$$
\Phi_{n+1}=\Phi_{n} L_{n}, \quad L_{n}=R_{1}\left(-\nu_{n+1}\right) R_{3}\left(\kappa_{n+1}\right),
$$

where

$$
R_{1}(\theta)=\left[\begin{array}{ccc}
1 & 0 & 0 \\
0 & \cos \theta & -\sin \theta \\
0 & \sin \theta & \cos \theta
\end{array}\right], \quad R_{3}(\theta)=\left[\begin{array}{ccc}
\cos \theta & -\sin \theta & 0 \\
\sin \theta & \cos \theta & 0 \\
0 & 0 & 1
\end{array}\right],
$$

and $\nu_{n}, \kappa_{n}$ are defined by

$$
\begin{gathered}
\left\langle T_{n-1}, T_{n}\right\rangle=\cos \kappa_{n}, \quad\left\langle B_{n}, B_{n-1}\right\rangle=\cos \nu_{n}, \quad\left\langle B_{n}, N_{n-1}\right\rangle=\sin \nu_{n}, \\
-\pi \leq \nu_{n}<\pi, \quad 0<\kappa_{n}<\pi .
\end{gathered}
$$

In order to formulate a "good" discrete deformation (discretization of time), we resort to the theory of discrete integrable systems to preserve integrable nature of the NLS flow (4). As a discrete analogue of NLS (9), we consider

$$
\begin{gathered}
\left(\sqrt{-1} \frac{\epsilon^{2}}{\delta}-1\right) u_{n}^{m+1}-\left(\sqrt{-1} \frac{\epsilon^{2}}{\delta}+1\right) u_{n}^{m}+\left(u_{n+1}^{m}+u_{n-1}^{m+1}\right)\left(1+\epsilon^{2}\left|u_{n}^{m}\right|^{2}\right) \Gamma_{n}^{m}=0, \\
\frac{\Gamma_{n+1}^{m}}{\Gamma_{n}^{m}}=\frac{1+\epsilon^{2}\left|u_{n}^{m}\right|^{2}}{1+\epsilon^{2}\left|u_{n}^{m+1}\right|^{2}},
\end{gathered}
$$

which we refer to as the discrete nonlinear Schrödinger equation (dNLS) $[1,2,7]$. Here, $u_{n}^{m} \in \mathbb{C}$, $\Gamma_{n}^{m} \in \mathbb{R}, n$ is the space discrete variable which corresponds to the label of vertices of discrete curves, $m$ is the discrete variable corresponding to the step of deformation, $\epsilon$ and $\delta$ are constants which are 
the lattice intervals of $n$ and $m$, respectively. Moreover, $u_{n}^{m}$ is the complex discrete curvature defined by

$$
u_{n}^{m}=\frac{1}{\epsilon} \tan \frac{\kappa_{n}^{m}}{2} e^{\sqrt{-1} \Lambda_{n}^{m}}, \quad \Lambda_{n}^{m}-\Lambda_{n-1}^{m}=-\nu_{n}^{m},
$$

We impose the boundary condition as

$$
u_{n}^{m} \rightarrow 0(n \rightarrow \pm \infty), \quad \Gamma_{n}^{m} \rightarrow \Gamma_{ \pm \infty} \text { (const.) }(n \rightarrow \pm \infty) .
$$

Then one of the main statements of this paper is given as follows:

\section{Theorem 1 (dNLS flow)}

For a fixed $m$, let $\gamma_{n}^{m} \in \mathbb{R}^{3}$ be a space discrete curve satisfying

$$
\left|\gamma_{n+1}^{m}-\gamma_{n}^{m}\right|=\epsilon,
$$

and $\Phi_{n}^{m}=\left[T_{n}^{m}, N_{n}^{m}, B_{n}^{m}\right] \in \mathrm{SO}(3)$ be the discrete Frenet frame defined in (11) satisfying the discrete Frenet-Serret formula

$$
\Phi_{n+1}^{m}=\Phi_{n}^{m} L_{n}^{m}, \quad L_{n}^{m}=R_{1}\left(-\nu_{n+1}^{m}\right) R_{3}\left(\kappa_{n+1}^{m}\right) .
$$

Let $u_{n}^{m}$ be a complex discrete curvature of $\gamma_{n}^{m}$. We determine $u_{n}^{m+1}$ by $d N L S$ (15) under the boundary condition (17) and put $u_{n}^{m+1}=\frac{1}{\epsilon} \tan \frac{\kappa_{n}^{m+1}}{2} e^{\sqrt{-1} \Lambda_{n}^{m+1}}$. We define a new curve $\gamma_{n}^{m+1} \in \mathbb{R}^{3}$ by

$$
\begin{gathered}
\frac{\gamma_{n}^{m+1}-\gamma_{n}^{m}}{\delta}=\frac{2}{\epsilon^{3}}\left(P_{n}^{m} T_{n}^{m}+Q_{n}^{m} N_{n}^{m}+R_{n}^{m} B_{n}^{m}\right), \\
P_{n}^{m}=\delta\left(-1+\frac{\Gamma_{n}^{m}}{\cos ^{2} \frac{\kappa_{n}^{m}}{2}}\right), \\
Q_{n}^{m}=\delta\left[\tan \frac{\kappa_{n}^{m}}{2}-\tan \frac{\kappa_{n-1}^{m+1}}{2} \cos \left(\Lambda_{n-1}^{m+1}-\Lambda_{n}^{m}\right) \frac{\Gamma_{n}^{m}}{\cos ^{2} \frac{\kappa_{n}^{m}}{2}}\right], \\
R_{n}^{m}=\epsilon^{2} \tan \frac{\kappa_{n}^{m}}{2}-\delta \tan \frac{\kappa_{n-1}^{m+1}}{2} \sin \left(\Lambda_{n-1}^{m+1}-\Lambda_{n}^{m}\right) \frac{\Gamma_{n}^{m}}{\cos ^{2} \frac{\kappa_{n}^{m}}{2}} .
\end{gathered}
$$

Suppose that $\Gamma_{\infty}=1$ or $1+\frac{\epsilon^{4}}{\delta^{2}}$. Then, it follows that

(1) $\left|\gamma_{n+1}^{m+1}-\gamma_{n}^{m+1}\right|=\epsilon$. Namely, $\gamma_{n}^{m+1}$ is an isoperimetric deformation of $\gamma_{n}^{m}$.

(2) $u_{n}^{m+1}$ gives the complex discrete curvature of $\gamma_{n}^{m+1}$.
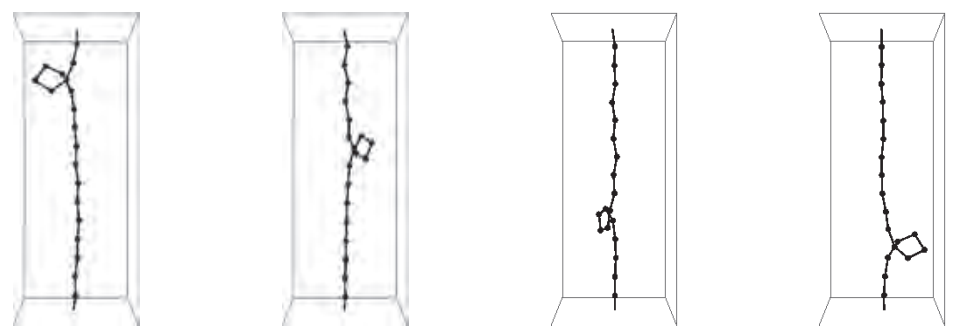

Figure 1: Numerical simulation of dNLS flow.

\section{Remark 2}


(1) The deformation (21) is not an equidistant deformation in contrast with the deformation described by dmKdV [13]. In fact, one can show that

$$
\left|\frac{\gamma_{n}^{m+1}-\gamma_{n}^{m}}{\delta}\right|^{2}=\frac{4}{\epsilon^{2}}\left(-1+\frac{\Gamma_{n}^{m}}{\cos ^{2} \frac{\kappa_{n}^{m}}{2}}\right) .
$$

Equation (22) also implies that the solution of dNLS (15) should satisfy the condition $\Gamma_{n}^{m} \geq$ $\cos ^{2} \frac{\kappa_{n}^{m}}{2}$ in order to be consistent with the curve deformation. Note that this property does not contradict with Hoffmann's formulation where the deformation is constructed as composition of two isoperimetric and equidistant deformations.

(2) Continuous limit with respect to time can be simply taken as $t=m \delta$ and $\delta \rightarrow 0$. Then dNLS (15) and corresponding deformation equation (20) and (21) yields the semi-discrete $N L S$ equation or the Ablowitz-Ladik equation $[1,2]$

$$
\sqrt{-1} \frac{d u_{n}}{d t}+\frac{u_{n+1}-2 u_{n}+u_{n-1}}{\epsilon^{2}}+\left(u_{n+1}+u_{n-1}\right)\left|u_{n}\right|^{2}=0,
$$

and the deformation equation of space discrete curves $[3,9,16]$

$$
\frac{d}{d t} \gamma_{n}=\frac{2}{\epsilon} \tan \frac{\kappa_{n}}{2} B_{n}
$$

The dNLS flow (20) and (21) implies the deformation of Frenet frame as

$$
\begin{aligned}
\Phi_{n}^{m+1} & =\Phi_{n}^{m} M_{n}^{m} \\
M_{n}^{m} & =\frac{1}{\Gamma_{n+1}^{m}}\left[\begin{array}{ccc}
\left|\alpha_{n}^{m}\right|^{2}-\left|\beta_{n}^{m}\right|^{2} & 2 \Re\left(\alpha_{n}^{m} \beta_{n}^{m *}\right) & -2 \Im\left(\alpha_{n}^{m} \beta_{n}^{m *}\right) \\
-2 \Re\left(\alpha_{n}^{m} \beta_{n}^{m}\right) & \Re\left(\alpha_{n}^{m 2}-\beta_{n}^{m 2}\right) & -\Im\left(\alpha_{n}^{m 2}+\beta_{n}^{m 2}\right) \\
-2 \Im\left(\alpha_{n}^{m} \beta_{n}^{m}\right) & \Im\left(\alpha_{n}^{m 2}-\beta_{n}^{m 2}\right) & \Re\left(\alpha_{n}^{m 2}+\beta_{n}^{m 2}\right)
\end{array}\right] \in \mathrm{SO}(3),
\end{aligned}
$$

where $\alpha_{n}^{m}, \beta_{n}^{m} \in \mathbb{C}$ are given by

$$
\begin{aligned}
\alpha_{n}^{m} & =\sqrt{-1} \frac{\delta}{\epsilon^{2}}\left[\left(1-\sqrt{-1} \frac{\epsilon^{2}}{\delta}\right)-\left(1+\epsilon^{2} u_{n+1}^{m} u_{n}^{m+1 *}\right) \Gamma_{n+1}^{m}\right] e^{\frac{\sqrt{-1}}{2}\left(\Lambda_{n}^{m+1}-\Lambda_{n}^{m}\right)}, \\
\beta_{n}^{m} & =\sqrt{-1} \frac{\delta}{\epsilon}\left(u_{n}^{m+1}-u_{n+1}^{m}\right) \Gamma_{n+1}^{m} e^{-\frac{\sqrt{-1}}{2}\left(\Lambda_{n}^{m}+\Lambda_{n}^{m+1}\right)},
\end{aligned}
$$

respectively. Here, $*$ means the complex conjugate. The Frenet-Serret formula (19) and the deformation equation (25) can be transformed to the $\mathrm{SU}(2)$ version by the standard correspondence of $\mathrm{SO}(3)$ and $\mathrm{SU}(2)$ as

$$
\begin{aligned}
& \phi_{n+1}^{m}=\phi_{n}^{m} L_{n}^{m}, \quad L_{n}^{m}=\left[\begin{array}{cc}
\cos \frac{\kappa_{n+1}^{m}}{2} e^{-\frac{\sqrt{-1}}{2} \nu_{n+1}^{m}} & -\sin \frac{\kappa_{n+1}^{m}}{2} e^{-\frac{\sqrt{-1}}{2} \nu_{n+1}^{m}} \\
\sin \frac{\kappa_{n+1}^{m}}{2} e^{\frac{\sqrt{-1}}{2} \nu_{n+1}^{m}} & \cos \frac{\kappa_{n+1}^{m}}{2} e^{\frac{\sqrt{-1}}{2} \nu_{n+1}^{m}}
\end{array}\right], \\
& \phi_{n}^{m+1}=\phi_{n}^{m} M_{n}^{m}, \quad M_{n}^{m}=\frac{1}{\sqrt{\Gamma_{n+1}^{m}}}\left[\begin{array}{cc}
\alpha_{n}^{m} & \beta_{n}^{m} \\
-\beta_{n}^{m *} & \alpha_{n}^{m *}
\end{array}\right],
\end{aligned}
$$

which is known as the Lax pair of dNLS $[1,2]$. In fact, one can verify that the compatibility condition $L_{n}^{m} M_{n+1}^{m}=M_{n}^{m} L_{n}^{m+1}$ yields dNLS (15).

\section{Outline of the proof of Theorem 1}

The first statement may be verified directly in principle, by computing $\gamma_{n+1}^{m+1}-\gamma_{n}^{m+1}$ and its length from (20), (21) and the discrete Frenet-Serret formula (19) under the assumption that $u_{n}^{m+1}$ is determined by dNLS (15). However, this computation is hopelessly complicated to carry out. To make it 
feasible, we change the Frenet frame to a different frame used in $[6,8]$, which we call the complex parallel frame in this paper. Let $F_{n}^{m}=\left[T_{n}^{m}, U_{n}^{m}, U_{n}^{m *}\right] \in \mathrm{U}(3)$ be the complex parallel frame defined by

$$
U_{n}^{m}=\frac{e^{\sqrt{-1} \Lambda_{n}^{m}}}{\sqrt{2}}\left(N_{n}^{m}+\sqrt{-1} B_{n}^{m}\right) .
$$

Note that it is related to the discrete Frenet frame $\Phi_{n}^{m}$ as

$$
F_{n}^{m}=\Phi_{n}^{m}\left[\begin{array}{ccc}
1 & 0 & 0 \\
0 & 1 & 1 \\
0 & \sqrt{-1} & -\sqrt{-1}
\end{array}\right]\left[\begin{array}{ccc}
1 & 0 & 0 \\
0 & \frac{e^{\sqrt{-1} \Lambda_{n}^{m}}}{\sqrt{2}} & 0 \\
0 & 0 & \frac{e^{-\sqrt{-1} \Lambda_{n}^{m}}}{\sqrt{2}}
\end{array}\right]
$$

Then the complex curvature $u_{n}^{m}$ naturally arises in this framework; the discrete Frenet-Serret formula (19) and the deformation of the discrete curve are rewritten in terms of $u_{n}^{m}$ as

$$
F_{n+1}^{m}=F_{n}^{m} X_{n}^{m}, X_{n}^{m}=\frac{1}{1+\epsilon^{2}\left|u_{n+1}^{m}\right|^{2}}\left[\begin{array}{ccc}
1-\epsilon^{2}\left|u_{n+1}^{m}\right|^{2} & -\sqrt{2} \epsilon u_{n+1}^{m}{ }^{*} & -\sqrt{2} \epsilon u_{n+1}^{m} \\
\sqrt{2} \epsilon u_{n+1}^{m} & 1 & -\epsilon^{2}\left(u_{n+1}^{m}\right)^{2} \\
\sqrt{2} \epsilon u_{n+1}^{m}{ }^{*} & -\epsilon^{2}\left(u_{n+1}^{m}{ }^{*}\right)^{2} & 1
\end{array}\right]
$$

and

$$
\gamma_{n}^{m+1}=\gamma_{n}^{m}+\frac{2 \delta^{2}}{\epsilon^{3}} F_{n}^{m}\left[\begin{array}{c}
-1+\left(1+\epsilon^{2}\left|u_{n}^{m}\right|^{2}\right) \Gamma_{n}^{m} \\
\frac{\epsilon}{\sqrt{2}}\left\{\left(1-\sqrt{-1} \frac{\epsilon^{2}}{\delta}\right) u_{n}^{m *}-u_{n-1}^{m+1 *}\left(1+\epsilon^{2}\left|u_{n}^{m}\right|^{2}\right) \Gamma_{n}^{m}\right\} \\
\frac{\epsilon}{\sqrt{2}}\left\{\left(1+\sqrt{-1} \frac{\epsilon^{2}}{\delta}\right) u_{n}^{m}-u_{n-1}^{m+1}\left(1+\epsilon^{2}\left|u_{n}^{m}\right|^{2}\right) \Gamma_{n}^{m}\right\}
\end{array}\right],
$$

respectively. The following lemma plays a crucial role in the proof:

Lemma 3 Under the boundary condition (17), it holds that

$$
\left|\alpha_{n}^{m}\right|^{2}+\left|\beta_{n}^{m}\right|^{2}=\Gamma_{n+1}^{m},
$$

provided that $\Gamma_{ \pm \infty}=1$ or $1+\frac{\epsilon^{4}}{\delta^{2}}$.

By using (32), we have after a long but straightforward calculations

$$
T_{n}^{m+1}=\frac{\gamma_{n+1}^{m}-\gamma_{n}^{m}}{\epsilon}=F_{n}^{m} \frac{1}{\Gamma_{n+1}^{m}}\left[\begin{array}{c}
\left|\alpha_{n}^{m}\right|^{2}-\left|\beta_{n}^{m}\right|^{2} \\
-\sqrt{2} \alpha_{n}^{m *} \beta_{n}^{m *} e^{-\sqrt{-1} \Lambda_{n}^{m}} \\
-\sqrt{2} \alpha_{n}^{m} \beta_{n}^{m} e^{\sqrt{-1} \Lambda_{n}^{m}}
\end{array}\right]
$$

from which we obtain

$$
\left|\frac{\gamma_{n+1}^{m+1}-\gamma_{n}^{m+1}}{\epsilon}\right|^{2}=\frac{\left(\left|\alpha_{n}^{m}\right|^{2}-\left|\beta_{n}^{m}\right|^{2}\right)^{2}+4\left|\alpha_{n}^{m}\right|^{2}\left|\beta_{n}^{m}\right|^{2}}{\Gamma_{n+1}^{m}{ }^{2}}=\left(\frac{\left|\alpha_{n}^{m}\right|^{2}+\left|\beta_{n}^{m}\right|^{2}}{\Gamma_{n+1}^{m}}\right)^{2}=1 .
$$

This proves the first statement. The second statement is proved as follows. Starting from $T_{n}^{m+1}$ (33), we have $B_{n}^{m+1}$ and $N_{n}^{m+1}$ in terms of $F_{n}^{m}$ by using (11). Then we obtain an expression of $\Phi_{n}^{m+1}=\left[T_{n}^{m+1}, N_{n}^{m+1}, B_{n}^{m+1}\right]$ in terms of $F_{n}^{m}$, which can be rewritten as $F_{n}^{m+1}=F_{n}^{m} Y_{n}^{m}$ with a certain matrix $Y_{n}^{m} \in \mathrm{U}(3)$ by using (29). This can be also transformed to the deformation equation of discrete Frenet frame of the form $\Phi_{n}^{m+1}=\Phi_{n}^{m} M_{n}^{m}$ with $M_{n}^{m}$ given in (25). Finally one can check that $\Phi_{n}^{m+1}$ satisfies the discrete Frenet-Serret formula (19) for $\kappa_{n}^{m+1}$ and $\nu_{n}^{m+1}$ determined from the complex curvature $u_{n}^{m+1}$. This completes the proof of Theorem 1. 


\section{Explicit formulas}

The formulation of NLS and dNLS flows in terms of the Frenet frame enables us to apply the theory of integrable systems. As an example, we here present explicit formulas of the NLS and dNLS flows in terms of the $\tau$ functions. For the case of plane curves, see [12]. These formulas are established based on the bilinear formalism in the theory of integrable systems by applying suitable reductions and imposing complex structure to $\tau$ functions of the 2-component KP hierarchy, but here we only show the results.

For any $N \in \mathbb{N}$, we first introduce the following three determinants, a $2 N \times 2 N$ determinant $\tau$, two $(2 N+1) \times(2 N+1)$ determinants $\sigma$ and $\rho$ as

$$
\tau=\left|\begin{array}{ccc:ccc}
m_{11}^{(1)} & \cdots & m_{1 N}^{(1)} & 1 & & \varnothing \\
\vdots & \cdots & \vdots & & \ddots & \\
m_{N 1}^{(1)} & \cdots & m_{N N}^{(1)} & \varnothing & & 1 \\
\hdashline-1 & & \varnothing & m_{11}^{(2)} & \cdots & m_{1 N}^{(2)} \\
& \ddots & & \vdots & \cdots & \vdots \\
\varnothing & & -1 & m_{N 1}^{(2)} & \cdots & m_{N N}^{(2)}
\end{array}\right|
$$

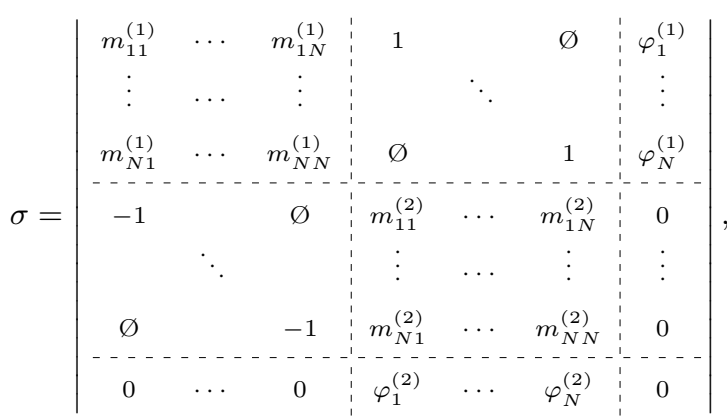

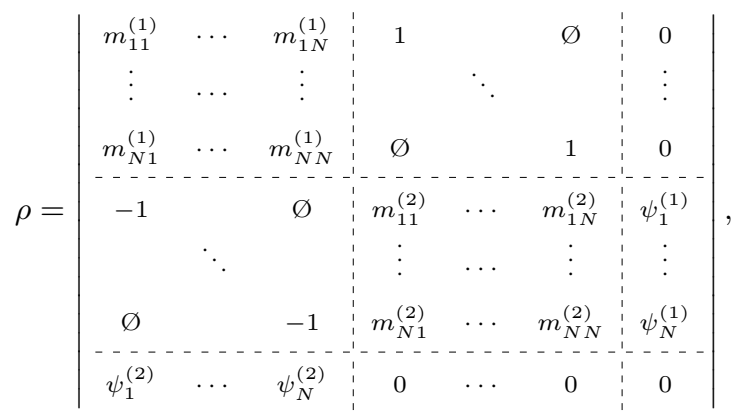

where $\varnothing$ is the empty block. Then the formulas for NLS flow on smooth curves and dNLS flow on discrete curves are obtained by choosing the entries of determinant as follows: 


\section{NLS flow on smooth curves}

We choose the entries of determinants as

$$
\begin{aligned}
& \varphi_{i}^{(1)}=p_{i}^{n} e^{\xi_{i}}, \quad \varphi_{i}^{(2)}=-1, \\
& \psi_{i}^{(1)}=1, \quad \psi_{i}^{(2)}=-\left(-\frac{1}{p_{i}^{*}}\right)^{n} e^{\xi_{j}^{*}}, \\
& m_{i j}^{(1)}=-\frac{\varphi_{i}^{(1)} \psi_{j}^{(2)}}{p_{i}+p_{j}^{*}}, \quad m_{i j}^{(2)}=\frac{1}{p_{i}^{*}+p_{j}}, \\
& \xi_{i}=p_{i} x-\sqrt{-1} p_{i}^{2} t+\frac{1}{p_{i}} z+\xi_{i}^{(0)}, \quad p_{i}, \xi_{i}^{(0)} \in \mathbb{C},
\end{aligned}
$$

so that we write $\tau=\tau_{n}(x, t ; z), \sigma=\sigma_{n}(x, t ; z), \rho=\rho_{n}(x, t ; z)$. Here, $n$ and $z$ are regarded as auxiliary variables. Putting

$$
F=\tau_{0}, \quad G=-\rho_{0}, \quad h=-\sigma_{-2},
$$

we have:

\section{Theorem 4 (Explicit formula for NLS flow) ${ }^{1}$}

(1) Let $u=u(x, t) \in \mathbb{C}$ be

$$
u=2 \frac{G}{F}
$$

Then u satisfies $N L S(9)$.

(2) Let $\gamma=\gamma(x, t) \in \mathbb{R}^{3}$ be

$$
\gamma=\left[\begin{array}{c}
\frac{h+h^{*}}{F} \\
\frac{1}{\sqrt{-1}} \frac{h-h^{*}}{F} \\
2 \frac{\partial}{\partial z}(\log F)-x
\end{array}\right] .
$$

Then $\gamma$ satisfies the Frenet-Serret formula (3) and the deformation equation (4).
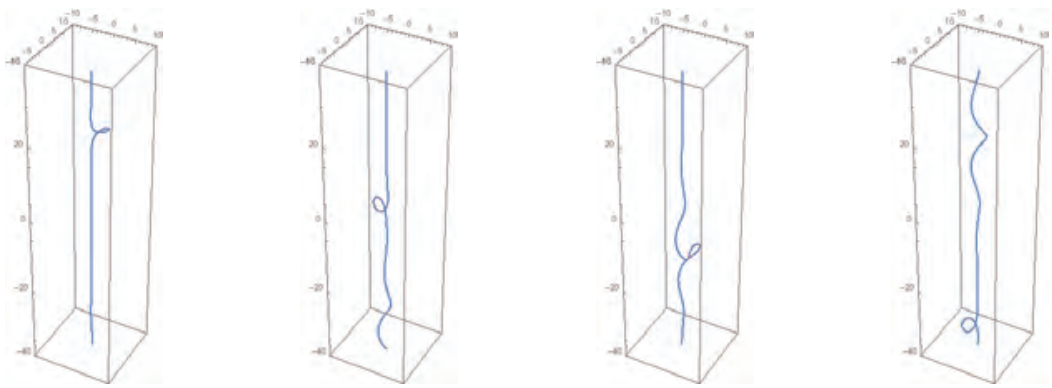

Figure 2: Interaction of loops of smooth curve by NLS flow obtained from Theorem 4.

\footnotetext{
${ }^{1}$ The $N$-soliton solution for the tangent vector has been constructed by using the bilinear formalism in [5].
} 


\section{dNLS flow on discrete curves}

We choose the entries of determinants as

$$
\begin{aligned}
& \varphi_{i}^{(1)}=p_{i}^{-n} e^{\zeta_{i}}\left(1-a p_{i}\right)^{-m}\left(1-c p_{i}\right)^{-r}, \quad \varphi_{i}^{(2)}=-\left(1-\frac{a}{p_{i}}\right)^{m}\left(1-\frac{1}{c p_{i}}\right)^{s}, \\
& \psi_{i}^{(1)}=\left(1-a p_{i}^{*}\right)^{-m}\left(1-\frac{p_{i}^{*}}{c}\right)^{-s}, \quad \psi_{i}^{(2)}=-\left(p_{i}^{*}\right)^{-n} e^{\zeta_{i}^{*}}\left(1-\frac{a}{p_{i}^{*}}\right)^{m}\left(1-\frac{c}{p_{i}^{*}}\right)^{r}, \\
& m_{i j}^{(1)}=-\frac{\varphi_{i}^{(1)} \psi_{j}^{(2)}}{p_{i}-\frac{1}{p_{j}^{*}}}, \quad m_{i j}^{(2)}=-\frac{\psi_{i}^{(1)} \varphi_{j}^{(2)}}{p_{i}^{*}-\frac{1}{p_{j}}}, \quad e^{\zeta_{i}}=e^{\frac{1}{2} \frac{1+c p_{i}}{1-c p_{i}} z}, \\
& a \in \mathbb{R}, \quad p_{i}, c \in \mathbb{C}, \quad|c|=1,
\end{aligned}
$$

so that we write $\tau=\tau_{n}^{m}(r, s ; z), \sigma=\sigma_{n}^{m}(r, s ; z), \rho=\rho_{n}^{m}(r, s ; z)$ with $r, s$ and $z$ being auxiliary variables. Putting

$$
\begin{gathered}
F_{n}^{m}=\tau_{n}^{m}(0,0 ; z), \quad G_{n}^{m}=\rho_{n}^{m}(0,0 ; z), \quad h_{n}^{m}=c^{-n} \sigma_{n}^{m}(1,-1 ; z), \\
a=\left(1+\frac{\epsilon^{4}}{\delta^{2}}\right)^{\frac{1}{2}}, \quad c=\frac{1-\sqrt{-1} \frac{\epsilon^{2}}{\delta}}{a},
\end{gathered}
$$

we have:

\section{Theorem 5 (Explicit formula for dNLS flow)}

(1) Let $u_{n}^{m} \in \mathbb{C}$ be

$$
u_{n}^{m}=\frac{(-1)^{m} c^{-n-2 m}}{\epsilon} \frac{G_{n}^{m}}{F_{n}^{m}}, \quad \Gamma_{n}^{m}=\frac{2 a}{c+\frac{1}{c}} \frac{F_{n-1}^{m+1} F_{n}^{m}}{F_{n}^{m+1} F_{n-1}^{m}} .
$$

Then $u_{n}^{m}$ satisfies dNLS (15).

(2) Let $\gamma_{n}^{m} \in \mathbb{R}^{3}$ be

$$
\gamma_{n}^{m}=\epsilon\left[\begin{array}{c}
(-1)^{m} \frac{h_{n}^{m}+h_{n}^{m *}}{F_{n}^{m}} \\
\frac{(-1)^{m}}{\sqrt{-1}} \frac{h_{n}^{m}-h_{n}^{m *}}{F_{n}^{m}} \\
2 \frac{\partial}{\partial z}\left(\log F_{n}^{m}\right)-n-2 m
\end{array}\right] .
$$

Then $\gamma_{n}^{m}$ satisfies the Frenet-Serret formula (19) and the deformation equation (20), (21).
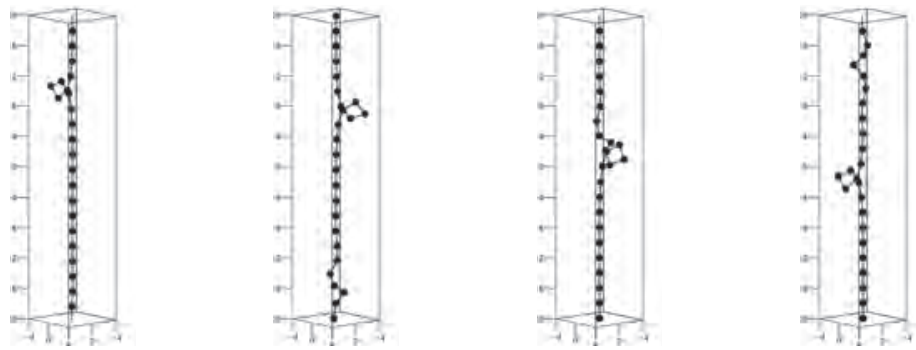

Figure 3: Interaction of loops of discrete curve by dNLS flow obtained from Theorem 5. 


\section{References}

[1] M.J. Ablowitz and J.F. Ladik, A nonlinear difference scheme and inverse scattering, Stud. in Appl. Math. 55(1976) 213-229.

[2] M.J. Ablowitz and J.F. Ladik, On the solution of a class of nonlinear partial difference equations, Stud. in Appl. Math. 57(1977) 1-12.

[3] A. Doliwa and P.M. Santini, Integrable dynamics of a discrete curve and the Ablowitz-Ladik hierarchy, J. Math. Phys. 36(1995) 1259-1273.

[4] A. Doliwa and P. M. Santini, The integrable dynamics of a discrete curve, Symmetries and Integrability of Difference Equations, D. Levi, L. Vinet and P. Winternitz (eds.), CRM Proceedings \& Lecture Notes Vol.9 (American Mathematical Society, Providence, RI, 1996) 91-102.

[5] Y. Fukumoto and T. Miyazaki, N-Solitons on a curved vortex filament, J. Phys. Soc. Jpn. 55(1986) 4152-4155.

[6] H. Hasimoto, Soliton on a vortex filament, J. Fluid Mech. 51(1972) 477-485.

[7] R. Hirota and S. Tsujimoto, Difference scheme of soliton equations, in: Future directions of nonlinear dynamics in physical and biological systems, P.L. Christiansen, J.C. Eilbeck and R.D. Parmentier (eds.), NATO ASI Series 312 (Springer 1993) 7-15.

[8] G.L. Lamb, Solitons on moving space curves, J. Math. Phys. 18(1977) 1654-1659.

[9] M. Hisakado and M. Wadati, Moving discrete curve and geometric phase, Phys. Lett. A214(1996) 252-258.

[10] T. Hoffmann, On the equivalence of the discrete nonlinear Schrödinger equation and the discrete isotropic Heisenberg magnet, Phys. Lett. A 265 (2000) 62-67.

[11] T. Hoffmann, Discrete Hashimoto surfaces and a doubly discrete smoke-ring flow, Discrete Differential Geometry, A.I. Bobenko, P. Schröder, J.M. Sullivan and G.M. Ziegler (eds.), Oberwolfach Seminars Vol.38 (Birkhäuser, Basel, 2008) 95-115.

[12] J. Inoguchi, K. Kajiwara, N. Matsuura and Y. Ohta, Motion and Bäcklund transformations of discrete plane curves, Kyushu J. Math. 66(2012) 303-324.

[13] J. Inoguchi, K. Kajiwara, N. Matsuura and Y. Ohta, Discrete $\mathrm{mKdV}$ and discrete sine-Gordon flows on discrete space curves, J. Phys. A: Math. Theor. 47(2014) 235202.

[14] J. Inoguchi, K. Kajiwara, N. Matsuura and Y. Ohta, Discrete isoperimetric deformation of discrete curves, in: Ken Anjyo (ed.), Mathematical progress in expressive image synthesis, Mathematics for Industry 4 (Springer 2014) 111-122.

[15] N. Matsuura, Discrete KdV and discrete modified KdV equations arising from motions of planar discrete curves, Int. Math. Res. Not. 2012(2012) 1681-1698.

[16] K. Nakayama, Elementary vortex filament model of the discrete nonlinear Schrödinger equation, J. Phys. Soc. Jpn. 76(2007) 074003.

[17] K. Nakayama, H. Segur and M. Wadati, Integrability and the motions of curves, Phys. Rev. Lett. 69(1992) 2603-2606.

[18] U. Pinkall, B. Springborn and S. Weißmann, A new doubly discrete analogue of smoke ring flow and the real time simulation of fluid flow, J. Phys. A: Math. Theor. 40 (2007) 12563-12576.

[19] S. Weißmann and U. Pinkall, Real-time interactive simulation of smoke using discrete integrable vortex filaments, in: H. Prautzsch et al.(eds.), Workshop on virtual reality interaction and physical simulations VRIPHYS (The Eurographics Association, 2009) 1-10. 